Schwächen auf, da sie weitgehend nur eine sehr geraffte Darstellung der Inhalte liefern.

Dafür bieten die Teile 3 und 4 einen sehr guten und ausführlichen Einblick in die Leistungen von ISIS bzw. GISIS. Über beide Systeme lassen sich Datenbankanfragen, die über sinnvolle Suchparameter (Sensor, Zeit, geographischer Ort) eingrenzbar sind, formulieren und Fernerkundungsdaten bestellen. Daneben verfügt das System über Serviceleistungen wie eine ausführliche Ergebnisliste, die Quicklooks gewährt, über Preise und Datentypen informiert und Adressen von Direktanbietern weitergibt. Zum Teil können Daten auch schon direkt transferiert werden. Außerdem kann man in ISIS z. B. über das Infoboard Hintergrundinformationen abfragen; dies ist auch mit GISIS möglich, obwohl das Infoboard hier anders aufgebaut ist. Ein Vergleich der beiden Systeme zeigt sehr schnell, daß GISIS für den Nutzer deutlich attraktiver ist, da die Recherche weniger umständlich und schneller als bei ISIS durchgeführt werden kann. Darüber hinaus verfügt GISIS über den Map Browser, der die Suche und Ergebnisanzeige erfolgreich unterstützt; z. B. kann man mit seiner Hilfe die Lage von Bildern anzeigen lassen, ohne diese mühsam über die Koordinatenangaben in den Ergebnislisten erschließen zu müssen.

Die Zusammenstellung in Teil 5 dient dem weniger versierten Nutzer als Hilfestellung, da hier die Eigenschaften der verfügbaren Basis- daten vorgestellt werden. Zusätzlich wird auf die Inhalte der beiliegenden CD verwiesen. Diese bietet eine Übersicht über die Standardprodukte des DFD, eine Auswahl thematisch aufbereiteter Daten, Beschreibungen von Verfahren zur Satellitenbildauswertung (im HTMLFormat) sowie Ausschnitte von Originaldaten zum Selbststudium (im BMP-Format). Dabei ist die vorgestellte Auswahl sehr anschaulich, praxisnah und ausführlich.

Zusammenfassend läßt sich feststellen, daß dem Nutzer ein umfassender Eindruck der Anwendungsmöglichkeiten von ISIS bzw. GISIS vermittelt wird. Die Erklärungen und Hinweise zur Durchführung der Recherche etc. sind leicht verständlich und nachvollziehbar. Dennoch ist der Buchtitel „ISIS - Fernerkundung für jedermann" letztendlich irreführend, da dem Nutzer einige Vorkenntnisse im Hard- und Softwarebereich und auf dem Gebiet der Fernerkundung abverlangt werden.

Christiane Lechtenbörger, Bochum

\section{Kartographie im Internet}

\section{$\square$ Society of Cartographers}

Eine interessante Homepage ist die der Society of Cartographers. Hier findet man eine Fülle von unterschiedlichen Informationen und Links zu weiteren Seiten. Für MacUser dürfte vor allem eine Seite interessant sein, auf der alle Vorteile des Einsatzes eines Macintosh auf- gelistet sind. Die Adresse der Society of Cartographers lautet: http://www.shef.ac.uk/uni/projects/ sc/index.html

$\square$ Kartographie + GIS im Internet http://www.hut.fi/TKK/Kartografia/ English/news/hotlist.html

http://www. seic.okstate.edu/serv.

html.

http://www. seic.okstate.edu/serv/

foreign.html

http://www.iko.unit.no/gis/gisen.

html

http://www.geo.ed.ac.uk/geoinfo/

dem.send

http://www.geo.de/geomap/

Homepage U.S. Geological Survey:

http://www.usgs.gov/

USGS Thematic Maps: http://cpg.

cr.usgs.gov/thmaps/thematic.htm

$\square$ Neuerdings steht ein Tutorial über GIS und Internetanwendungen für jedermann bereit. Das Tutorial basiert auf einer privaten Initiative und wird kostenfrei über das Internet zur Verfügung gestellt: URL = http://www.bonn.netsurf.de/gistutor oder http://www.ginb.uni-bonn.de/ gistutor. Es ist mit und ohne direkten Internet-Zugang einsetzbar. Voraussetzung ist lediglich eine installierte Browser-Software wie Netscape Navigator, MS-Explorer, Mosaic oder andere. Eine Offline-Version für MS-Windows kann für eine $\mathrm{Be}$ arbeitungsgebühr von 19,50 DM (Selbstkosten inkl. Porto) unter folgender Kontaktadresse bestellt werden: Dipl.-Ing. Roland Stahl, Meisenstr. 2, D-53859 Niederkassel, Tel.: 0228 / 454464, E-mail: Roland.Stahl@bonn.netsurf.de.

\title{
Veranstalfungen 1997
}

\section{7. -19.9 \\ 22. $-26,9$. \\ 81. Geodätentag/Intergeo in Karlsnuhe; Leitthema; "Technik für Raum und Recht* \\ 46. Photogrammetrische Woche in Stuttgart, Institut für Photogrammetrie der Univer- sitât (s. KN 1/97, S. 27 f.)}

$29.9,-2.10$

6. 10.

$6,-11.10$.

7.- 8.10

7. -10.10

21. Arbeitskurs Niederdollendorf in Königslutter, als Amwenderfonum ,Digitale Kartentechnologie" des AK Praktische Kartagraphie (s. KN 2/97, S. 76 u. 77)

Festkollogium „40 Jahre Kartographieausbildung an der TU Dresden*, Institut für Kartographie, TU Dresden (s. KN 2/97, S. 73)

51. Deutscher Geographentag in Bonn; Leitthema: "Europa in einer Welt im Wandel* 34. Sitzung der Arbeitsgruppe „Automation in der Kanographie" (AGA). Instiut fü Kartographie, Technische Universität Dresden (s. KN 2197) ring, Tel. $089 / 894343-0$ 